\title{
Anticoagulação, Gravidez e Cardiopatia. Uma Tríade, três Domínios e cinco Momentos
}

\author{
Walkiria Samuel Avila, Max Grinberg \\ São Paulo, SP
}

Os recentes consensos em muito contribuíram para minimizar potenciais riscos obstétricos e fetais e melhorar a assistência a um número cada vez maior de pacientes durante o ciclo gravídicopuerperal. Anticoagulação versus gravidez é uma associação que, freqüentemente, suscita dilema à beira do leito, vez que significa reprogramar a fisiologia da coagulação em busca do ponto de equilíbrio entre os riscos de tromboembolismo materno e os de hemorragia, inerente ao anticoagulante, conjugado aos diversos momentos da gestação, do parto e do puerpério. Acrescente-se, ainda, o efeito teratogênico da varfarina sódica.

As considerações que se seguem são reflexões sobre o tema, baseadas na significativa experiência do Setor de Cardiopatia e Gravidez da Unidade Clínica de Valvopatias do InCor.

\section{Risco tromboembólico e obrigatoriedade da anticoagulação}

O potencial tromboembólico está centrado, habitualmente, nas circunstâncias antecedentes e seguintes à concepção, com grande possibilidade de agravamento devido às particularidades inerentes à gravidez, como as reológicas, mecânicas e hormonais, entre outras (quadro I). Há duas modalidades de anticoagulação a ser consideradas durante o ciclo gravídico-puerperal, com respeito ao período de uso:

1. Anticoagulação permanente é recomendada às mulheres que apresentam condições trombogênicas pré-gestacionais e cujo benefício supera sobejamente os riscos (quadro II). Próteses mecânicas cardíacas e fibrilação atrial são as indicações mais freqüentes de anticoagulação permanente correspondendo, respectivamente, a 38 e $32 \%$ das gestantes com cardiopatia, sob anticoagulação, no InCor. Tromboembolismo e complicações hemorrágicas constituem as maiores desvantagens das próteses mecânicas durante a gravidez, entretanto os índices de complicações tromboembólicas de 3,9 a $33,3 \%^{1}$ e a taxa de mortalidade materna de 1,8 a $4,2 \%^{1}$ exigem a anticoagulação permanente e fundamentam as normas de conduta. Análise de 35 gestantes usuárias de anticoagulantes e portadoras de próteses mecânicas, no InCor mostrou que sete (20\%) delas apresentaram acidente tromboembólico sendo quatro casos de trombose de prótese e três de episódio isquêmico

Incor do Hospital das Clínicas da FMUSP.

Endereço para Correspondência: Dr. Max Grinberg. Av. Dr. Eneas de Carvalho Aguiar, 44 - Cep 05403-000 - São Paulo - SP transitório, reforçando o alto risco de tromboembolismo na gestação ${ }^{2}$. Por sua vez, a incidência de hemorragia, estimada em $2,5 \%{ }^{1}$, está relacionada em $80 \%$ dos casos ao parto e a doenças obstétricas nem sempre evitáveis, como abortamento espontâneo, placenta prévia, descolamento prematuro de placenta e parto prematuro. A fibrilação atrial, por ser evento habitual na história natural das valvopatias reumáticas, é situação clínica observada com freqüência na gestante cardiopata ${ }^{3}$. Embora a gravidez seja um período relativamente curto, o estado de hipercoagulabilidade materna favorece o aumento das taxas de embolia.

2. Anticoagulação transitória é indicada em estados clínicos inexistentes antes da gestação (quadro II). Neste grupo destacam-se, pela freqüência, a trombose venosa profunda e o tromboembolismo pulmonar, causas de morbimortalidade materna, estimadas em 1 óbito para 1.500 partos $^{4}$. A embolia pulmonar incide em aproximadamente $16 \%$ das pacientes com trombose venosa profunda não tratada, sendo que $2 / 3$ dos eventos acontecem durante a gestação e o restante nas primeiras semanas após o parto. Também não é rara, durante a gravidez, a cardioversão elétrica, sob anticoagulação transitória, para reverter ao ritmo sinusal a fibrilação ou o flutter atrial, sendo esta considerada o método de escolha por ser mais eficiente, abreviar o período de instabilidade hemodinâmica materna e evitar a administração de outros fármacos. A ocorrência de tromboembolismo em pacientes com fibrilação atrial submetidos a cardioversão sem ter recebido anticoagulante prévio é estimada entre 1 e 7\%, taxa que se reduz a menos de $1 \%$ quando a cardioversão é realizada sob efeito de anticoagulação ${ }^{5}$. A indicação de anticoagulação transitória para pacientes submetidas a repouso hospitalar prolongado é assunto controverso a despeito da presença de situações predisponentes ao tromboembolismo, como cardiopatias congênitas cianóticas, hipertensão arterial pulmonar e insuficiência cardíaca.

\section{Peculiaridades dos anticoagulantes no ciclo gravídico-puerperal}

O uso da varfarina sódica apresenta riscos peculiares ao feto, com graus variáveis a cada trimestre. Ela atravessa a barreira placentária e é teratogênica, particularmente entre a $6^{\mathrm{a}}$ e a $9^{\mathrm{a}}$ semana de gestação, acarretando 1 a 3\% de malformações congênitas caracterizadas pela síndrome varfarínico-fetal, e favorece o abortamento espontâneo em cerca de 10 a 33\%. Embora o percentual de incidência seja controverso, a varfarina pode acarretar anormalidades no sistema nervoso central quando usada no $2^{\circ}$ 
trimestre da gestação e hemorragia meníngea, por compressão do pólo cefálico fetal, no período expulsivo do parto ${ }^{6}$. No puerpério, os estudos disponíveis não conseguiram detectar níveis de varfarina sódica no leite materno nem no sangue de lactentes presumivelmente expostos a esse medicamento pelo aleitamento natural, assim como também não encontraram alteração no perfil de coagulação dessas crianças? ${ }^{7}$.

A heparina não-fracionada (HNF) e a heparina de baixo peso molecular (HBPM) não atravessam a barreira placentária e por isto são, conceitualmente, os anticoagulantes preferenciais durante a gravidez. Contudo, seu uso prolongado associa-se a efeitos colaterais maternos, incluindo trombocitopenia ${ }^{8}$, hemorragia e osteoporose $^{9}$. As vantagens da HBPM sobre a HNF são a superioridade quanto à biodisponibilidade (100\% vs 30\%), à vida média após a aplicação (2h vs até $60 \mathrm{~min}$ ), à absorção por via subcutânea (100\% vs variável) e à menor incidência de trombocitopenia $(0 \%$ vs $2,7 \%)^{10,11}$. Contudo, limitações ao seu uso incluem a neutralização inadequada pelo sulfato de protamina e o alto custo.

\begin{tabular}{|ll|}
\hline Quadro I - Fatores da gravidez que predispõem ao tromboembolismo \\
\hline Reológico & - Aumento na concentração dos fatores V, VI, VII, IX e X \\
& - Aumento da atividade plaquetária \\
& - Aumento da síntese do fibrinogênio \\
Mecânico & - Redução do retorno venoso \\
Hormonal & - Modificação da complacência arterial \\
Outros & - Parto cesárea \\
& - Obesidade \\
& - Repouso prolongado \\
& - Pré-eclampsia \\
& - Infecções \\
\hline
\end{tabular}

\begin{tabular}{|c|c|c|}
\hline \multicolumn{3}{|c|}{ Quadro II - Principais indicações cardíacas de anticoagulação na gravidez } \\
\hline Permanente & $\begin{array}{l}\text { Alto risco } \\
\text { Menor risco }\end{array}$ & $\begin{array}{l}\text { - Prótese valvar mecânica } \\
\text { - Fibrilação atrial permanente } \\
\text { - Antecedente tromboembólico }\end{array}$ \\
\hline Transitória & $\begin{array}{l}\text { Obrigatória } \\
\text { Discutível }\end{array}$ & $\begin{array}{l}\text { - Trombose venosa profunda: tratamento } \\
\text { e prevenção } \\
\text { - Cardioversão elétrica } \\
\text { - Cardiomiopatia dilatada } \\
\text { - Insuficiência cardíaca congestiva } \\
\text { - Cardiopatias congênitas cianóticas } \\
\text { - Trombo intracavitário } \\
\text { - Repouso prolongado }\end{array}$ \\
\hline
\end{tabular}

\section{Recomendações, controle e eficácia da anticoagulação}

Não há consenso sobre qual seria o melhor esquema de anticoagulação para as gestantes face os diversos riscos, presumidos, de trombose, porque nenhuma opção farmacológica, isolada ou combinada, forneceu fortes evidências quanto à eficácia em não acarretar efeitos colaterais à mãe e/ou ao feto.

A experiência multidisciplinar do Hospital das Clínicas da Faculdade de Medicina da Universidade de São Paulo fundamentou a elaboração de Recomendações para a prevenção do tromboembolismo na gestação de mulher cardiopata ${ }^{12}$, sumarizadas no quadro III. Elas pretendem, com apoio na literatura ${ }^{13-15}$, atender aos requisitos ideais das diretrizes, como: credibilidade, baseada no conhecimento científico; validade, em acordo com os melhores resultados; reprodutibilidade, efetiva em distintos serviços; e, representatividade, fundamentada na vivência dos autores. Ressalve-se que, como qualquer diretriz, sua aplicação exige flexibilidade, que é a adaptação às circunstâncias de cada caso.

Os estudos clínicos continuam apoiando o emprego da varfarina na anticoagulação permanente para portadoras de próteses mecânicas e desencorajando o uso da HNF subcutânea ${ }^{16-20}$. Dados do InCor reforçam os da literatura ao mostrarem que $85 \%$ dos casos de tromboembolismo durante a gravidez de portadoras de próteses mecânicas associaram-se ao uso de HNF em alguma fase da gestaçãa ${ }^{2,21}$. Admite-se que a ineficácia da HNF subcutânea, nesta situação, deve-se, particularmente, às dificuldades de obtenção da adequada anticoagulação, de seu controle e de aderência da paciente ao medicamento.

Nos casos de fibrilação atrial permanente tem sido recomendada a anticoagulação em doses menores de heparina ou de varfarina ajustadas ao coagulograma (quadro III).

A HBPM ganhou espaço na anticoagulação transitória de pacientes com risco menor e está, gradativamente, tomando o lugar da HNF subcutânea também para pacientes de alto risco, como nos casos de próteses mecânicas ${ }^{19}$. Nessas condições, embora haja escassez de estudos, seu uso tem sido recomendado em doses terapêuticas controladas pelo fator anti-Xa (quadro III). A propósito, o consenso norte-americano sobre anticoagulação de pacientes grávidas portadoras de próteses valvares, publicado em

\begin{tabular}{|c|c|c|c|c|}
\hline \multicolumn{5}{|c|}{ Quadro III - Recomendações, doses e controle } \\
\hline Permamente & Anticoagulante & Via & Dose & Controle \\
\hline Prótese mecânica & $\begin{array}{l}\text { HNF } \\
\text { Enoxaparina } \\
\text { Varfarina }\end{array}$ & $\begin{array}{l}\text { IV } \\
\text { SC } \\
\text { VO }\end{array}$ & $\begin{array}{l}18 U \mathrm{U} / \mathrm{kg} / \mathrm{h} \\
1 \mathrm{mg} / \mathrm{kg} \text { cada } 12 \mathrm{~h} \\
\text { Ajustada }\end{array}$ & $\begin{array}{l}\text { TTPA }=1,5 \text { a } 2,0 \text { vezes vn } \\
\text { Fator anti-Xa }-0,6 \text { a } 1,0 \\
\text { INR }-2,5 \text { a } 3,0\end{array}$ \\
\hline Fibrilação atrial & $\begin{array}{l}\text { HNF } \\
\text { Enoxaparina } \\
\text { Varfarina }\end{array}$ & $\begin{array}{l}\text { SC } \\
\text { SC } \\
\text { VO }\end{array}$ & $\begin{array}{l}10-20.000 \text { UI cada } 12 \mathrm{~h} \\
1 \mathrm{mg} / \mathrm{kg} / \mathrm{dia} \\
\text { ajustada }\end{array}$ & $\begin{array}{l}\text { TTPA } \leq 1,5, \text { a } 2 \text { vezes } v n \\
\text { Fator anti-Xa }-0,3 \text { a } 0,6 \\
\text { INR }-2,0\end{array}$ \\
\hline $\begin{array}{l}\text { Transitória } \\
\text { Obrigatória }\end{array}$ & $\begin{array}{l}\text { HNF } \\
\text { Enoxaparina } \\
\text { Enoxaparina }\end{array}$ & $\begin{array}{l}\text { IV } \\
\text { SC } \\
\text { SC }\end{array}$ & $\begin{array}{l}18 U \mathrm{Ul} / \mathrm{kg} / \mathrm{h} \\
1 \mathrm{mg} / \mathrm{kg} \text { cada } 12 \mathrm{~h} \\
1 \mathrm{mg} / \mathrm{kg} / \mathrm{dia}\end{array}$ & $\begin{array}{l}\text { TTPA }=1,5 \text { a } 2,0 \text { vezes } v n \\
\text { Fator anti-Xa }-0.6 \text { a } 1.0\end{array}$ \\
\hline Discutível & $\begin{array}{l}\text { HNF } \\
\text { Enoxaparina }\end{array}$ & $\begin{array}{l}\text { SC } \\
\text { SC }\end{array}$ & $\begin{array}{l}10 \text { a } 20.000 \text { UI/dia } \\
1 \mathrm{mg} / \mathrm{kg} / \mathrm{dia}\end{array}$ & TTPA $\leq 1,5$ a 2,0 vezes $v n$ \\
\hline
\end{tabular}

HNF - heparina não-fracionada; TTPA - tempo de tromboplastina parcial ativada; vn - valor normal; INR - International Normalized Ratio; IV - intravenoso; SC - subcutâneo; VO - via oral 
$2002^{13}$, concluiu que a enoxaparina deve ser uma opção terapêutica durante o período gestacional e considerou as informações relatadas na bula do produto (Aventis ${ }^{\circledR}$ ) como inconsistentes com as experiências clínicas favoráveis, dados institucionais e ensaios comparativos com a HNF em portadoras de próteses mecânicas.

A anticoagulação transitória nos casos de trombose venosa profunda tem sido recomendada com o emprego da HNF intravenosa ou HBPM, em doses ajustadas ao coagulograma, no período de cinco a sete dias, e posterior manutenção em doses menores, subcutânea, até o final do puerpério ${ }^{14}$. Quando a cardioversão elétrica for realizada no $1^{\circ}$ trimestre recomenda-se a HNF ou a HBPM subcutânea, escolhendo-se a varfarina para os demais trimestres, em doses ajustadas ao coagulograma (quadro III). $\mathrm{O}$ fármaco (heparina ou varfarina) deve ser administrado três semanas antes e mantido três semanas após a reversão ao ritmo sinusal. Finalmente, para a anticoagulação transitória em casos discutíveis, com risco presumivelmente menor (quadro II) pela ausência de fatores tromboembólicos, recomenda-se o uso de HNF ou HBPM em doses menores (quadro III).

Estas normas têm sido aplicadas no InCor sob o raciocínio de fragmentação obstétrica em cinco momentos: planejamento familiar; cada trimestre; e, parto e puerpério, estratégia esta que se revelou útil para reflexões pedagógicas sobre a dinâmica da anticoagulação.

\section{Momento I}

Planejamento familiar (pré-gestação): conscientização da paciente/casal, capacidade da equipe médica, avaliação dos recursos de infra-estrutura.

O momento I diz respeito, essencialmente, à paciente que faz uso permanente de anticoagulante oral. Utilizando a linguagem bioética ${ }^{22,23}$, o ponto essencial é o respeito à autonomia da paciente. Deve-se, portanto,destacar que não há contra-indicação à gestação mas alertar para a potencial morbidade do uso da varfarina sódica. Outrossim, obriga-se o estabelecimento ao compromisso médico-paciente em função das variáveis previsíveis: risco de hemorragia, tromboembolismo, abortamento, teratogênese, hospitalização prolongada, custos e riscos da administração parenteral do anticoagulante. 0 diálogo franco com o casal é fundamental. A conscientização de paciente/casal transita pela exatidão das informações médicas, o reforço sobre o porquê do uso da anticoagulação, as opções farmacológicas, a aderência aos esquemas terapêuticos, a atenção ao pronto diagnóstico da gravidez e a mobilização para imediato desencadeamento da estratégia de ação já no primeiro trimestre. A capacitação da equipe médica inclui a contrapartida do conhecimento sobre o benefício/malefício de cada opção farmacêutica, o senso clínico para diagnosticar precocemente a gravidez e a agilidade para adaptar o esquema de anticoagulação de oral para parenteral. A preocupação com a disponibilidade de infra-estrutura alicerça-se nos controles laboratoriais devido às mudanças de condutas: INR (International Normalized Ratio) e fator anti-Xa, dosagem da gonadotrofina coriônica beta à primeira dúvida em função de atraso menstrual, hospitalização prolongada para heparinização intravenosa. Também é desejável a participa-

\section{Momento II}

Primeiro trimestre: evitar teratogênese, substituir o anticoagulante, controlar a anticoagulação, evitar hemorragia.

O ponto fundamental neste momento II é a substituição da anticoagulação permanente, oral, pela parenteral. Do ponto de vista da bioética ${ }^{22,24}$, há preocupação em manter o benefício da anticoagulação para a condição mórbida da paciente e evitar o malefício da síndrome varfarínica. A conciliação entre esses dois pólos aponta para a recomendação do uso da heparina. Concordamos com a literatura que a HBPM fornece maiores recursos para a otimização da anticoagulação, associando praticidade e segurança, em relação à HNF, no decorrer desse trimestre inicial. Idealmente, a partir do resultado positivo do teste de gonadotrofina coriônica ( $\beta \mathrm{HCG}$ ), faz-se a substituição da varfarina sódica pela enoxaparina, em uso permanente, com doses ajustadas de acordo com o coagulograma nas situações de alto risco e em doses menores nas de menor risco (quadro III). A paciente deve receber informações objetivas de como proceder diante da eventualidade de sangramento vaginal, hematoma espontâneo ou dor no baixo ventre. A administração da HNF (intravenosa, em pacientes de alto risco, e subcutânea, em doses menores, para as demais situações) é motivo de discussão na literatura ${ }^{1}$. Para as portadoras de próteses mecânicas os riscos correm por conta da trombocitopenia induzida pela heparina com conseqüente trombose, hospitalização prolongada, flebite e bacteriemia.

\section{Momento III}

Segundo trimestre: retornar ao anticoagulante oral, controlar a anticoagulação, evitar hemorragia.

Nesse momento III, a liberação para o retorno da varfarina sódica conjuga-se com a pressuposição de se reduzir os efeitos adversos à mãe, ao se abreviar o tempo de uso de heparina. Desaparece o impedimento da varfarina em vista do menor risco de teratogenicidade do momento II, e reaparece a lógica do período pré-gestacional, ou seja, a preferência pela anticoagulação oral. Aspecto de importância quando da re-introdução da varfarina é o ajuste da dose sob rigorosa vigilância dos níveis de INR. Há necessidade da "dinâmica de transição" ${ }^{24}$, ou seja: manter o benefício da HBPM, enquanto são aguardados os valores de INR admitidos como de proteção antitrombose. Em princípio, a transição pode ser desfeita quando o INR, em ascensão, atingir valor de $1,8-2,0$

\section{Momento IV}

Terceiro trimestre: redirecionar para a anticoagulação parenteral, controlar a anticoagulação, evitar hemorragia, planejar o parto, considerar a hospitalização.

Ao contrário dos trimestres anteriores em que a conduta era homogênea, respectivamente, só heparina no $1^{\circ}$ e só varfarina no $2^{\circ}$, o $3^{\circ}$ trimestre caracteriza-se por uma atitude de transição: suspensão da varfarina e introdução da heparina ${ }^{19}$. A conduta outrora tomada de substituir a varfarina pela heparina 15 dias antes da data provável do parto associa-se a questões como antecipações de eventos naturais como parto prematuro e exposição, tanto da 
mãe como do feto, à hemorragia. Por isso, é pertinente considerar como benéfica a substituição da varfarina a partir da $36^{\mathrm{a}}$ semana de gestação, lembrando-se que o feto adquire maturidade após 37 semanas. No momento IV consideramos que a HNF intravenosa tem indicação nas pacientes de alto risco e a subcutânea, em doses menores, naquelas com menor risco (quadro III). Vida média mais curta e disponibilidade de fármaco-antagonista são as grandes vantagens conceituais da HNF sobre a HBPM, especialmente perante parto prematuro ou outras complicações obstétricas, acrescentando-se, ainda, maior autonomia quanto às decisões obstétricas e anestésicas sobre o parto. A hospitalização das pacientes de alto risco nas últimas quatro semanas de gestação justifica-se pela necessidade de administração intravenosa da HNF, de controles diários da anticoagulação e da saúde fetal, e de planejamento do parto.

\section{Momento V}

\section{Parto e puerpério: adequar o parto e a anestesia, adequar a anticoagulação parenteral, reintroduzir a anticoagulação oral.}

A discussão sobre o tipo de parto deve ser iniciada o mais precocemente possível quando se está face a gestante em uso de anticoagulante. Essa decisão pode ser tomada no início do $3^{\circ}$ trimestre baseando-se exclusivamente nas indicações obstétricas, contudo, na grande maioria dos casos, a indicação do tipo de parto acontece próximo ao termo da gestação. 0 cardiologista entende que para pacientes com anticoagulação permanente o parto vaginal é o mais pertinente e a anticoagulação é uma variável de peso entre os itens a serem considerados nessa decisão. Reconhece-se, entretanto, que os fatores obstétricos são hierarquicamente superiores no que tange à segurança tanto da mãe como do feto. A suspensão da anticoagulação deve integrar-se à dinâmica do parto e às decisões da equipe obstétrica com o objetivo de, nem deixar de suspender e nem prolongar o período sem anticoagulante. A anticoagulação restringe o uso da anestesia regional como a peridural ou a raquidiana devido aos riscos de hematoma extradural e compressão medular ${ }^{14}$. Entretanto, a suspensão da anticoagulação al- gumas horas antes do parto ( 4 a 24h), na dependência do esquema utilizado, pode permitir a aplicação da punção espinhal. A analgesia para o trabalho de parto e o parto vaginal deve obedecer às normas de cada serviço de acordo com a conduta convencional, contudo, deve acontecer na ausência de anticoagulante. Nas pacientes de alto risco e anticoagulação permanente, a suspensão da HNF intravenosa 4 a 6h antes do início da analgesia e da indução do parto tem proporcionado relativa segurança à punção medular e eficácia no parto vaginal. Para aquelas pacientes de menor risco e submetidas a menor dose de anticoagulação, a HNF ou a HBPM pode ser suspensa no dia anterior à indução do parto. Deve-se considerar que a cesárea, quando indicada, eleva o risco de hemorragia materna, restringe a reintrodução da anticoagulação plena em função do pós-operatório imediato e se associa a maior incidência de fenômenos tromboembólicos. O puerpério caracteriza-se pela fase crítica do estado de hipercoagulabilidade materno que, por si só, predispõe ao tromboembolismo. Por isto, decorridas $6 \mathrm{~h}$ do parto e em ausência de complicação obstétrica materna, a HNF intravenosa deve ser re-administrada às pacientes de alto risco; a HNF ou HBPM, em doses menores, nas demais situações; enquanto que a varfarina somente após 48h (quadro III). A suspensão da anticoagulação parenteral será concomitante ao alcance do INR adequado para cada caso. A amamentação não deve ser desestimulada em função da anticoagulação, uma vez que são insuficientes as informações disponíveis que suscitem sua contra-indicação.

0 retorno às condições pré-gestacionais costuma transcorrer à semelhança do que se observa no início da anticoagulação oral, ou seja, com heterogeneidade de comportamento ${ }^{5}$. Nesta fase, a paciente pode ter uma certa dificuldade em cumprir a rotina para estabilização da anticoagulação oral devido ao acúmulo de atividades como mãe. O papel do cardiologista é conscientizá-la de que estar adequadamente anticoagulada representa condição essencial para o cumprimento de sua responsabilidade materna.

\section{Agradecimentos}

À Dra Maéve de Barros Correia, pela revisão do texto.

\section{Referências}

1. Chan W S, Anand S, Gisberg J. Anticoagulation of pregnant women with mechanical heart valves: A systematic review of the literature. Arch Intern Med. 2000; 160:191-1962

2. Avila WS, Carvalho ME, Gouveia AMM, Reis CM, Grinberg M, Ramires JAF. Pregnancy in women with valve prostheses. Comparative study. Summary in Circulation. 2002; 1(Suppl II) : 525.

3. Avila WS, Rossi, EG, Ramires JAF et al. Pregnancy in patients with heart disease: Experience with 1,000 cases. Clin Cardiol. 2003 26,135-42.

4. Toglia MR, Weg JG. Current Concepts: Venous thromboembolism during pregnancy. N Engl J Med. 1996; 335 :108-14.

5. Arnold AZ, Mick MJ, Mazurek RP, Loop FD, Trohman RG. Role of prophylactic anticoagulation for direct current cardioversion in patients with atrial fibrillation or atrial flutter. J Am Coll Cardiol. 1992;19:851-5.

6. Hall JAG, Paul RM, Wilson KM. Maternal and fetal sequelae of anticoagulation during pregnancy. Am J Med. 1980;68:122-40.

7. Clark SL, Porter F, West FG. Coumarin derivates and breast-feeding. Obst \& Gynecol. 2000, 95, 6: 938-40.

8. Gruel Y. Heparin induced thrombocytopenia. Pathogenesis, diagnosis and treatment. Ver Med Interne. 2004 25:35-45

9. Ruiz-Irastorza G, Khamashita MA, Hughes GR. Heparin and osteoporosis during pregnancy. 2002 update. Lupus. 2002, 11:680-2.
10. Gould MK, Dembitzer AD, Doyle RL, Hostie TJ, Garber AM. Low molecular weight heparins compared with unfractionated heparin for treatment of acute deep venous thrombosis: a meta-analysis of randomized, controlled trials. Ann Intern Med. 1999; 130:800-9.

11. Dolovich L, Gisberg JS, Douketis JD, Holbrook AM, Cheah G. A meta-analysis comparing low molecular weight heparins to unfractionated heparin in the treatment of venous thromboembolism: examing some unanswered questions regarding location of treatment product type and dosing frequency. Arch Intern Med. 2000;160: 181-8.

12. Gouveia $\mathrm{AMM}$, Reis $\mathrm{CM}$, Cavalheiro $\mathrm{F}^{\circ} \mathrm{C}$ et al. Diretrizes de Anticoagulação na Gravidez. Boletim do Departamento de Cardiopatia e Gravidez da SBC. 2003; 10:

13. Topol EJ, Bosker G, Casele $\mathrm{H}$ et al. Anticoagulation and enoxaparina use in patients with prosthethic heart valves and/or pregnancy. Clinical Cardiol Consensus Report. 2002; 3: 1-18.

14. Gisberg J, Greer I, Hirsh J. Use of antithrombotic agents during pregnancy. Chest. 2001;11: 122S-31S.

15. Obstetric Medicine Group of Australasia. Anticoagulation in pregnancy and the puerperium. MJA. 2001; 175:258-63.

16. Golby AJ, Bush EC, DeRook FA, Albers GW. Failure of high dose heparin to prevent recurrent cardioembolic strokes in pregnant patient with mechanical heart valve. Neurology. 1992;42:2204-6. 
17. Watson WJ, Freeman J, O'Brien C, Benson M. Embolic stroke in a pregnant patient with a mechanical heart valve on optimal heparin therapy. Am J Perinatol. 1996;36:371-2.

18. Hurwitz A, Mildwidsky A, Medina A, Yagel ST. Failure of continuous intravenous heparinization to prevent stroke in a pregnant woman with a prosthetic valve and atrial fibrillation. J Reprod Med. 1985;30:618-20.

19. Sanson BJ, Lensing AW, Prins MH et al. Safety of low molecular weight heparin in pregnancy: a systematic review. Thromb Haemost.1999;81:668-72.

20. Arnaout MS, Kazma H, Khalil A et al. Is there a safe anticoagulation protocol for pregnant women with prosthetic valves? Clin Exp Obstet Gynecol. 1998;25101-4
21. Avila WS, Grinberg M, Martinelli Filho M, Snitcowsky R, Gutierrez, OS. Embolia coronária em gestante portadora de prótese de disco em posição aórtica. Relato de caso. Arq. Bras. Cardiol. 1985; 45:267-69.

22. Ribeiro RMD, Grinberg M, Spina GS et al. Consulta coletiva como ferramenta de orientação a pacientes em anticoagulação oral. Rev Soc Cardiol ESP. 2003;13:84

23. Grinberg M, Cohen C. Falando com o coração, auscultando a bioética. Rev Soc Cardiol ESP. 2002;6:805-20

24. Grinberg M. Entendo \& Aceito\& Faço. Arq Bras Cardiol. 2004; 82:309-12. 